\title{
Qubit Teleportation and Transfer across Antiferromagnetic Spin Chains
}

\author{
L. Campos Venuti, ${ }^{1,2}$ C. Degli Esposti Boschi, ${ }^{3,4}$ and M. Roncaglia ${ }^{4,2}$ \\ ${ }^{1}$ Institute for Scientific Interchange (ISI), Villa Gualino, viale Settimio Severo 65, I-10133 Torino, Italy \\ ${ }^{2}$ INFN Sezione di Bologna, viale C. Berti-Pichat 6/2, I-40127 Bologna, Italy \\ ${ }^{3}$ CNR, Unità CNISM di Bologna, viale C. Berti-Pichat 6/2, I-40127 Bologna, Italy \\ ${ }^{4}$ Dipartimento di Fisica, Università di Bologna, viale C. Berti-Pichat 6/2, I-40127 Bologna, Italy
}

(Dated: October 3, 2018)

\begin{abstract}
We explore the capability of spin- $1 / 2$ chains to act as quantum channels for both teleportation and transfer of qubits. Exploiting the emergence of long-distance entanglement in low-dimensional systems [Phys. Rev. Lett. 96, 247206 (2006)], here we show how to obtain high communication fidelities between distant parties. An investigation of protocols of teleportation and state transfer is presented, in the realistic situation where temperature is included. Basing our setup on antiferromagnetic rotationally invariant systems, both protocols are represented by pure depolarizing channels. We propose a scheme where channel fidelity close to one can be achieved on very long chains at moderately small temperature.
\end{abstract}

PACS numbers: 03.65.Ud, 03.67.Hk, 75.10.Pq

Introduction. In order to accomplish the main tasks of Quantum Information, a sizable amount of entanglement is needed [1]. In addition, the particles that share entanglement must be accessed individually for measurements and, quite importantly, they must be well separated in space.

Recently it was shown 2] that in some spin models at zero temperature (i.e. in the ground state) a selected pair of distant sites $A$ and $B$ can be highly entangled. In some cases sites $A$ and $B$ may be taken infinitely far apart still retaining a high amount of entanglement, a situation that was termed long distance entanglement (LDE). An example of this situation is given by the end-sites of an open $S=1 / 2$ dimerized Heisenberg chain. Even for moderate values of the dimerization this effect is strong enough to develop non local correlations, i.e. entanglement, between the end-sites of an open chain of infinite length.

The main aim of this Letter is to explore the actual feasibility of quantum teleportation and transfer across spin $1 / 2$ chains that exhibit LDE. Having in mind realistic optical lattice implementations of spin chains [3], we consider the principal cause of decoherence which is given by the temperature. Using the same schemes proposed in Ref. 2], we expect the entanglement between $A$ and $B$ to deteriorate when the temperature becomes of the order of the lowest excitation gap $\Delta$. As this gap, which originates from the boundary conditions, typically vanishes when the length $L$ of the chain increases, we are led to explore the tradeoff between temperature and chain length.

As will be clarified throughout this paper, antiferromagnetic chains with global SU(2)-invariance have several advantages. Typically, in these systems rotational symmetry is never broken. As a consequence the twoparticle reduced density matrix $\rho_{A B}$ (obtained by tracing the total $\rho$ over all the Hilbert space except sites $A$ and $B)$ maintains $\mathrm{SU}(2)$ invariance, i.e. it is a Werner state [4] in the language of quantum information. Werner states are described by a single parameter which can be taken to be $\left\langle\sigma_{A}^{z} \sigma_{B}^{z}\right\rangle_{\rho}=\operatorname{Tr}\left(\rho_{A B} \sigma_{A}^{z} \sigma_{B}^{z}\right) \in[-1,1 / 3]$. The interval $\left\langle\sigma_{A}^{z} \sigma_{B}^{z}\right\rangle_{\rho} \in[-1,-1 / 3)$ corresponds to entangled $\rho_{A B}$.

At $T=0$ the density matrix is $\rho=|G\rangle\langle G|$, with $|G\rangle$ the ground state, while at finite temperature it is given by the canonical density operator $\rho=Z^{-1} e^{-\beta H}$, with $\beta=1 / T$ (in units of $k_{B}$ ) and $Z$ the normalization factor. At low temperatures we can approximate the thermal density matrix by retaining only the ground state and the first excited states. On quite general grounds [5] the ground state $|G\rangle$ is a total singlet, while the first excitations are given by a spin one triplet $|m\rangle$ labeled by the total magnetization: $S_{\text {tot }}^{z}=m=-1,0,1$. Then at low temperatures we can write

$$
e^{-\beta H} \simeq e^{-\beta E_{0}}\left[|G\rangle\left\langle G\left|+e^{-\beta \Delta} \sum_{m=-1,0,1}\right| m\right\rangle\langle m|\right],
$$

where $E_{0}$ is the ground state energy and $\Delta$ is the first excitation gap. Notice that this approximation correctly maintains rotational invariance. The thermal reduced density matrix $\rho_{A B}(T)$ of $A$ and $B$ depends only on the following average value

$$
\begin{aligned}
\left\langle\sigma_{A}^{z} \sigma_{B}^{z}\right\rangle_{T}= & \frac{1}{1+3 e^{-\beta \Delta}}\left[\left\langle G\left|\sigma_{A}^{z} \sigma_{B}^{z}\right| G\right\rangle\right. \\
& \left.+e^{-\beta \Delta}\left(\left\langle 1\left|\sigma_{A}^{z} \sigma_{B}^{z}\right| 1\right\rangle+2\left\langle 1\left|\sigma_{A}^{x} \sigma_{B}^{x}\right| 1\right\rangle\right)\right],
\end{aligned}
$$

which has been written exploiting the $\mathrm{SU}(2)$ invariance. The form (2) is particularly useful for numerical densitymatrix renormalization-group (DMRG) simulations [6] since it involves only the computation of the lowest-state correlation functions in the sectors $m=0$ and $m=1$.

In the situations analyzed in 2] where LDE is present in the ground state, the $S=1$ triplet state is localized near the sites $A$ and $B$. As we will show below, the entan- 
glement in $\rho_{A B}(T)$ is maintained until $T$ becomes comparable with the gap $\Delta$, when the triplet state becomes non-negligible. We are then led to prefer systems with a large gap $\Delta$. Quite generally however, open systems with a finite bulk correlation length $\xi$ develop mid-gap levels scaling exponentially with the system size $\Delta \simeq e^{-L / \xi}$ 7]. On the other hand, systems with a diverging correlation length give rise to an algebraic decay, $\Delta \sim L^{-\alpha}$. The generality of this conjecture - that establishes a relation between bulk correlation length and the decay of the mid-gap - is a challenging question that deserves further studies.

For the above-mentioned reasons we propose to use an open $S=1 / 2$ Heisenberg chain with different interactions at the endpoints

$$
H_{\text {chain }}=H_{C}+J_{p}\left(\vec{S}_{A} \cdot \vec{S}_{2}+\vec{S}_{B} \cdot \vec{S}_{L-1}\right)
$$

as depicted in Fig. 1 (system $A C B$ ). In such a system there is strictly no LDE in the thermodynamic limit, but for finite size one can always choose $J_{p} / J$ small enough so as to have arbitrarily large entanglement between $A$ and $B$ in the ground state. Moreover we checked numerically that in this system the first gap $\Delta$ scales only algebraically with the size of the system $L: \Delta \sim L^{-\alpha}$ as can be seen in Fig. 2. Note the slow decay of the gaps due to the small value of $\alpha$ (see inset).

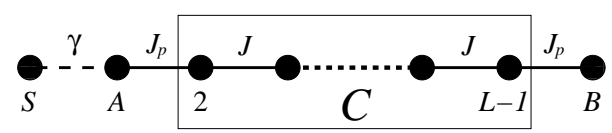

Figure 1: Model Hamiltonian considered for teleportation (joint measure between $S$ and $A$ ) and for transfer (switching on $\gamma$ at a given time).

Teleportation. Entangled Werner (SU(2) invariant) states have several advantages when used as a resource for quantum informational devices. As far as teleportation is concerned, one can show [8] that the standard teleportation scheme [9] is the best over all possible schemes at least in the region where a better-than-classical fidelity is achieved. In the standard protocol an unknown state $\xi$ at site $S$ (see Fig. 1) is teleported to site $B$ by making a joint Bell measurement on sites $S$ and $A$ and transmitting the result of the measurement $j$ to $B$ where a unitary transformation is applied. If $A$ and $B$ share a pure maximally entangled (SU(2) invariant) state $\left|\psi^{-}\right\rangle_{A B}=\left(|\uparrow \downarrow\rangle_{A B}-|\downarrow \uparrow\rangle_{A B}\right) / \sqrt{2}$, then the state $\xi$ is transferred to $B$ exactly. In a realistic situation, external noise of any kind turns the pure state $\left|\psi^{-}\right\rangle_{A B}$ into a non maximally entangled mixed state $\rho_{A B}$.

Using this protocol with a Werner state as resource, the fidelity of teleportation does not depend on the the outcome $j$ nor on the state to be teleported. By repeating the experiment many times with the same input state, the teleportation process is represented by a quantum

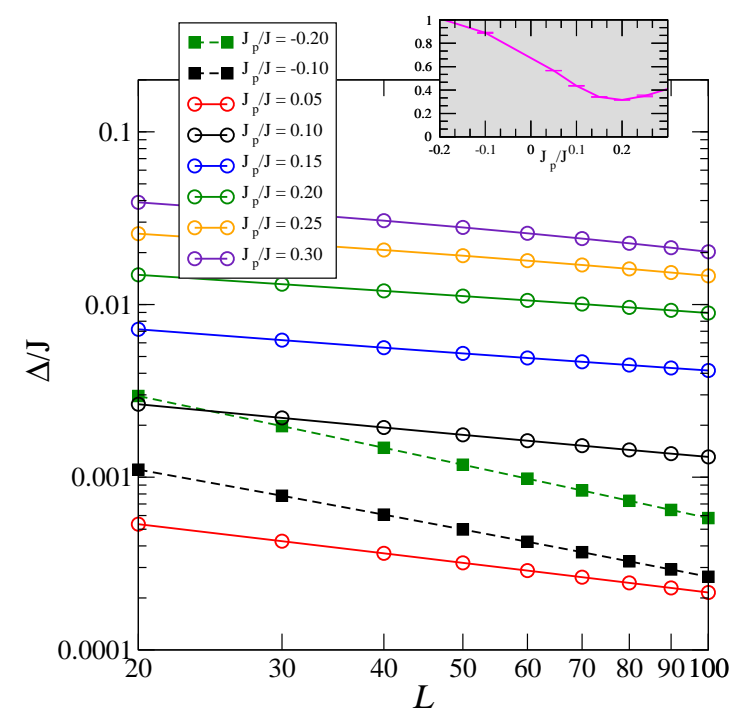

Figure 2: Finite size scaling behavior of the lowest gaps. In the inset is plotted the scaling exponent fitted with the law $\Delta=c L^{-\alpha}$. The data were obtained with a DMRG code using 400-500 optimized states and three finite system sweeps.

channel mapping input states $\xi$ at site $S$ into teleported states $\Lambda(\xi)$ at site $B[10]$. In this case, the teleportation channel is given precisely by a pure depolarizing channel:

$$
\Lambda(\xi)=\vartheta \xi+(1-\vartheta) \frac{1}{2} \mathbb{I} .
$$

The parameter $\vartheta$ which identifies the channel - sometimes called shrinking factor - takes the simple form $\vartheta=-\left\langle\sigma_{A}^{z} \sigma_{B}^{z}\right\rangle$. Obviously, $\Lambda$ turns into an ideal channel when $\vartheta=1$, i.e. when $\rho_{A B}$ is the singlet $\left|\psi^{-}\right\rangle_{A B}$. The fidelity of teleportation is

$$
f=\operatorname{Tr}(\xi \Lambda(\xi))=\frac{1+\vartheta}{2}=\frac{1-\left\langle\sigma_{A}^{z} \sigma_{B}^{z}\right\rangle}{2},
$$

that indeed does not depend on the state to teleport.

For our class of systems, $\vartheta$ is given by Eq. (2). When the temperature is increased from zero, it eventually reaches a value $T^{*}$, above which the thermal state $\rho_{A B}(T)$ becomes separable. This occurs when $\left\langle\sigma_{A}^{z} \sigma_{B}^{z}\right\rangle_{T^{*}}=-1 / 3$, that gives

$$
T^{*}=\Delta\left[\log \left(\frac{\left\langle 1\left|\sigma_{A}^{z} \sigma_{B}^{z}\right| 1\right\rangle+2\left\langle 1\left|\sigma_{A}^{x} \sigma_{B}^{x}\right| 1\right\rangle+1}{-\left\langle G\left|\sigma_{A}^{z} \sigma_{B}^{z}\right| G\right\rangle-1 / 3}\right)\right]^{-1} .
$$

Typical values in our scheme are obtained using the two qubit singlet and triplet pure states, for which we get $T^{*}=\Delta / \log (3) \approx 0.9 \Delta$. The gap $\Delta$ and the correlations appearing in Eq. (5) can be calculated numerically as functions of $L$ and $J_{p}$. In Fig. 3 we plot the results, obtained from DMRG simulations for a chain of $L=$ 50 sites. In view of an optical lattice experiment, these curves could serve to locate the working point to achieve the maximal possible fidelity. 


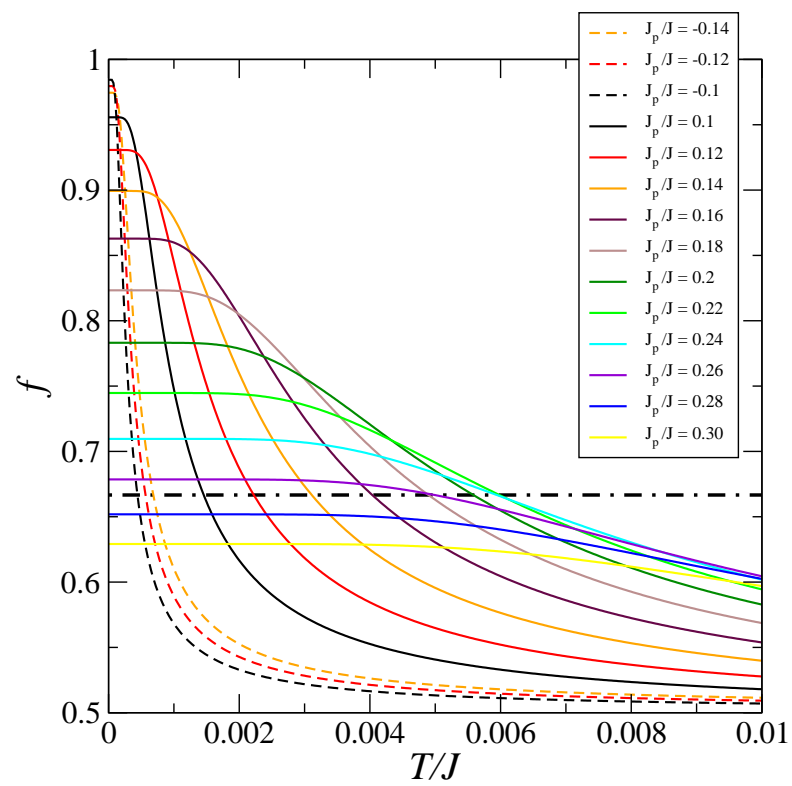

Figure 3: Fidelity of teleportation between end sites $A$ and $B$ as a function of temperature for the Heisenberg model. The curves refer to different values of the interaction $J_{p}$.

State transfer. As suggested by S. Bose [11] open spin chains can be exploited for transferring quantum states from one end to the other end of the chain. Let a chain of length $L$ be described by the Hamiltonian $H_{\text {chain }}$. For times $t<0$ the chain is in its ground state or possibly in a state of thermal equilibrium $\rho_{\text {chain }}=Z^{-1} e^{-\beta H_{\text {chain }}}$. At a time $t=0$ a spin-spin interaction $\gamma \vec{S}_{S} \cdot \vec{S}_{A}$ between the sender $S$ (that stores the pure state to be transferred $|\xi\rangle)$ and site $A$ is switched on and let to evolve with the Hamiltonian, as depicted in Fig. 1]

$$
H=H_{\text {chain }}+\gamma \vec{S}_{S} \cdot \vec{S}_{A}
$$

After a given optimal time $t^{*}$, the initial state $|\xi\rangle$ gets transferred to site $B$ with fidelity $f$.

We stress here the importance of dealing with antiferromagnetic interactions. In this case, elementary excitations typically have relativistic linear dispersion for small momenta, i.e. $\omega(k) \simeq v|k|$ where $v$ is the effective speed of light. On the contrary, in ferromagnetic systems, as the one originally proposed in [11], the dispersion of elementary excitations is generally quadratic for small momenta. This fact leads to dispersive effects which limit the fidelity of transfer.

From a quantum information perspective, one can easily show that the state transfer protocol with SU(2) invariant systems is precisely given by the depolarizing channel given by (4). The unique parameter specifying the channel is given in this case by $\vartheta=\left\langle\sigma_{B}^{z}(t)\right\rangle_{\rho}$, $\rho=|\uparrow\rangle\langle\uparrow| \otimes \rho_{\text {chain }}$, where the time evolution is according to the total Hamiltonian (6). The calculation of this quantity in a strongly correlated system is a non-trivial task. However, an approximation scheme is possible for the models where we observed LDE (or quasi-LDE).

Although the spins on $A$ and $B$ do not interact directly, they experience an effective interaction mediated by the system $C$. Due to rotational invariance, the model (3) is effectively mapped, at every perturbative order, onto an $\mathrm{SU}(2)$-symmetric Hamiltonian for the sites $A$ and $B$

$$
H_{\mathrm{eff}}=J_{\mathrm{eff}} \vec{S}_{A} \cdot \vec{S}_{B}
$$

This approximation holds when the energy splitting $J_{\text {eff }}$ caused by $H_{\text {eff }}$ is smaller than the typical gaps in the unperturbed Hamiltonian $H_{C}$. On the one hand, we know from conformal field theory [12] that finite-size gaps in $H_{C}$ scale as $J L^{-1}$. On the other hand, $J_{\text {eff }}$ is nothing but the singlet-triplet gap $\Delta$. We have numerically checked that $\Delta$ scales as $J L^{-\alpha}$, in the system $H_{\text {chain }}$, as can be seen from Fig. 2. The correct prefactor has the form $\Phi\left(J_{p} / J\right)$. From perturbation theory we know that $\Phi(x) \approx x^{2}$ for small $x$. This means that we can reliably approximate the model (3) with the effective Hamiltonian (7), provided that $\Phi\left(J_{p} / J\right)<L^{\alpha-1}$, i.e. $J_{p}<J L^{(\alpha-1) / 2}$ when $J_{p}$ is small enough.

Our scheme of approximation reduces the state transfer protocol to an effective three site problem where the time evolution is unitary by means of the Hamiltonian $H=\gamma \vec{S}_{S} \cdot \vec{S}_{A}+J_{\text {eff }} \vec{S}_{A} \cdot \vec{S}_{B}$. The average is done with respect to the ensemble $\rho_{0}=|\xi\rangle\left\langle\left.\xi\right|_{S} \otimes \rho_{A B}\right.$, where $\rho_{A B}$ is the most general mixed state which preserve $\mathrm{SU}(2)$ invariance, i.e.

$$
\rho_{A B}=\frac{1}{4} \mathbb{I}+\frac{g}{4} \vec{\sigma}_{A} \cdot \vec{\sigma}_{B},
$$

and $g=\left\langle\sigma_{A}^{z} \sigma_{B}^{z}\right\rangle$ that includes decoherence effects from the environment $C$ as well as the effect of temperature.

The density matrix evolves as

$$
\rho(t)=e^{-i t H}\left(|\xi\rangle\left\langle\left.\xi\right|_{S} \otimes \rho_{A B}\right) e^{i t H} .\right.
$$

The fidelity of the transfer from site $S$ to site $B$ at a given time $t$ is $f(t)=\operatorname{Tr}_{S A B}\left(\rho(t)|\xi\rangle\left\langle\left.\xi\right|_{B}\right)\right.$. After some calculations we get

$$
\begin{aligned}
f(t) & =\frac{1}{36 \omega^{2}}\left\{(22+4 g)\left(J_{\mathrm{eff}}^{2}+\gamma^{2}\right)-\gamma J_{\mathrm{eff}}(19+10 g)\right. \\
& -2(1+g) \omega\left[\omega_{-} \cos \left(t \omega_{+} / 2\right)+\omega_{+} \cos \left(t \omega_{-} / 2\right)\right] \\
& \left.+3 \gamma J_{\mathrm{eff}}(2 g-1) \cos (\omega t)\right\}
\end{aligned}
$$

where $\omega_{ \pm}=\omega \pm\left(J_{\text {eff }}+\gamma\right)$ and $\omega=\sqrt{J_{\text {eff }}^{2}-J_{\text {eff }} \gamma+\gamma^{2}}$. The maximal possible interference (constructive and destructive) is achieved when the frequencies are commensurate each other i.e. for $\gamma=J_{\text {eff }}$. In this case the fidelity reduces to

$$
\begin{aligned}
f(t) & =\frac{1}{36}\left[25-2 g-6(1+g) \cos \left(J_{\mathrm{eff}} t / 2\right)\right. \\
& \left.+(6 g-3) \cos \left(J_{\mathrm{eff}} t\right)+2(1+g) \cos \left(3 J_{\mathrm{eff}} t / 2\right)\right] .
\end{aligned}
$$


The first maximum of this function is attained at a time

$$
\begin{aligned}
t^{*} & =\frac{2}{J_{\mathrm{eff}}} \arccos \left(\frac{1-2 g-\sqrt{12 g^{2}+12 g+9}}{4(1+g)}\right) \\
& =\frac{\pi}{J_{\mathrm{eff}}}+\frac{2}{3} \frac{(g+1)}{J_{\mathrm{eff}}}+O\left((g+1)^{2}\right) .
\end{aligned}
$$

The value $g=-1$ represents the ideal case where we have a pure singlet $\rho_{A B}=\left|\psi^{-}\right\rangle\left\langle\left.\psi^{-}\right|_{A B}\right.$ at our disposal, with $t^{*}=\pi / \omega$. In the non-ideal case, the time for best transfer gets only slightly shifted by a value which in the worst case $(g=1 / 3)$ is 1.448 . The maximum fidelity is then

$$
\begin{aligned}
f^{*} & =f\left(t^{*}\right)=\frac{\sqrt{3}\left(4 g^{2}+4 g+3\right)^{3 / 2}+24 g^{2}+66 g+33}{48(1+g)^{2}} \\
& =1-\frac{2}{9}(g+1)+\frac{1}{18}(g+1)^{2}+O\left((g+1)^{3}\right) \cdot(10)
\end{aligned}
$$

As expected, the transfer is perfect for $g=-1$. However, the transfer fidelity remains very high for all the possible values $g \in[-1,1 / 3]$. The lowest possible value $f^{*}=7 / 8$ is attained at $g=0$ (maximally mixed case). Anyway, we must restrict ourself to the situation where the approximation of unitary evolution is valid, i.e. $g \simeq-1$, and correspondingly the transmission fidelity is very close to 1. As in the teleportation case, we have considered the transfer of a state using a Heisenberg chain playing the role of system $C$. In Fig. 4 the optimal transfer fidelity is plotted as a function of the chain length $L$ at temperature $T=0$ and $T=10^{-3} \mathrm{~J}$, for some values of $J_{p}$. In any case, we find a more-than-classical transmission fidelity even for chains of length 100 sites. For obtaining these results, the existence of entanglement between the two distant sites $A$ and $B$ is crucial .

Now, let us draw an additional consideration. First, we note that $t^{*} \propto J_{\text {eff }}^{-1}=L^{\alpha} /(J \Phi)$, with $\alpha<1$ (see Fig. 2) On the other hand, our scheme is expected to be valid under the condition $\Phi\left(J_{p} / J\right) \lesssim L^{\alpha-1}$ which implies $t^{*} \gtrsim$ $L / J$, consistently with the "flying" qubit picture where the information is carried by elementary spin excitations.

Finally, we mention that the transfer protocol may be used also for sharing entanglement between distant parties [11]. In our situation, we already have entanglement between distant parties $A$ and $B$, but we can ask how it may be further increased.

The idea is to start having a maximally entangled singlet $\rho_{\text {in }}=\left|\psi^{-}\right\rangle\left\langle\left.\psi^{-}\right|_{X S}\right.$ at sites $S$ and at an extra neighboring site $X$ completely decoupled from the rest. Then, we send the $S$ part of the input state $\rho_{\text {in }}$ through the quantum channel described by our transmission protocol. At a certain time $t^{*}$, we obtain an outcome state living on the pair of sites $X$ and $B$

$$
\rho_{\text {out }}=(1-p) \rho_{\text {in }}+\frac{p}{3} \sum_{k=1}^{3} \mathbb{I} \otimes \sigma^{k} \rho_{\text {in }} \mathbb{I} \otimes \sigma^{k},
$$

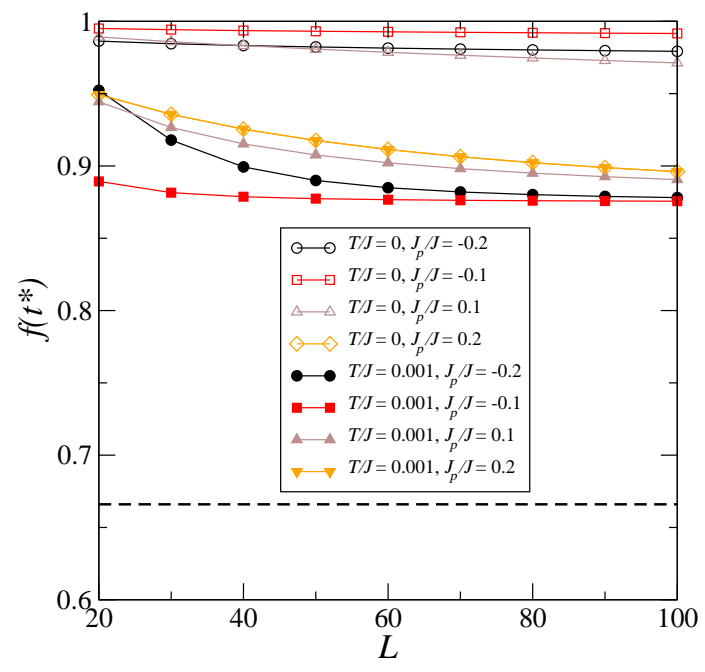

Figure 4: Transfer fidelity at optimal time as a function of the chain length $L$. The curves refer to some values the coupling $J_{p}$ between the probes $(A$ and $B)$ and the Heisenberg chain. Results are reported at both zero and finite temperature.

where $p=3(1-\vartheta) / 4$ is the so called error probability. In this state, the concurrence between $X$ and $B$ is

$$
C\left(\rho_{\text {out }}\right)=\max [1-2 p, 0]=\max \left[3 f^{*}-2,0\right],
$$

while original state $\rho_{A B}$ had a concurrence given by $C\left(\rho_{A B}\right)=\max [-3 / 2 g-1 / 2,0]$. Using $f^{*}$ from Eq. (10) it is possible to estimate that the concurrence is increased, i.e. $C\left(\rho_{\text {out }}\right) \geq C\left(\rho_{A B}\right)$, where the equality holds only when $g=-1$ (it is not possible to increase the entanglement of a singlet). The minimum value is achieved for the completely mixed case $g=0$ where the concurrence is $C\left(\rho_{\text {out }}\right)=5 / 8=0.625$.

Conclusions. We have given an explicit evidence that open antiferromagnetic Heisenberg chains may represent good quantum channels for teleportation and state transfer. This result relies mainly on the possibility to entangle the two end-spins (quasi-LDE) by choosing an appropriate coupling $J_{p}$. We have shown that, despite the smallness of the lowest gap, high fidelities of both teleportation and transfer may be achieved, with a tradeoff between temperature and chain length. It is tempting to speculate about the possibility of reproducing these effects in optical lattice environments 13].

We thank J.I. Cirac, M. Giampaolo, F. Illuminati, M. Keyl, D. Porras for interesting discussions and G. Morandi for a careful reading of the manuscript. This work was partially supported by the COFIN projects 2002024522_001 and 2003029498_013.

[1] M. A. Nielsen and I. L. Chuang, Quantum Computation and Quantum Information (Cambridge University Press, 
2000).

[2] L. Campos Venuti, C. Degli Esposti Boschi, and M. Roncaglia, Phys. Rev. Lett. 96, 247206 (2006).

[3] W. Hofstetter, Phil. Mag. 86, 1891 (2006).

[4] R. F. Werner, Phys. Rev. A 40, 4277 (1989).

[5] E. Lieb and D. C. Mattis, J. Math. Phys. 3, 749 (1962).

[6] U. Schollwöck, Rev. Mod. Phys. 77, 259 (2005).

[7] S. R. White and D. A. Huse, Phys. Rev. B 48, 3844 (1993).

[8] M. Horodecki, P. Horodecki, and R. Horodecki, Phys. Rev. A 60, 1888 (1999).
[9] C. H. Bennett, G. Brassard, C. Crépeau, R. Jozsa, A. Peres, and W. K. Wootters, Phys. Rev. Lett. 70, 1895 (1993).

[10] G. Bowen and S. Bose, Phys. Rev. Lett. 87, 267901 (2001).

[11] S. Bose, Phys. Rev. Lett. 91, 207901 (2003).

[12] M. Henkel, Conformal Invariance and Critical Phenomena (Springer, 1999).

[13] L. Campos Venuti, M. Giampaolo, and F. Illuminati (in preparation). 